\title{
CD103 - CD11b + dendritic cells regulate the sensitivity of CD4 T-cell responses to bacterial flagellin
}

\author{
SM Atif ${ }^{1}$, S Uematsu ${ }^{2}$, S Akira ${ }^{2}$ and SJ McSorley ${ }^{1}$
}

Toll-like receptor 5 (TLR5) has been widely studied in an inflammatory context, but the effect of TLR5 on the adaptive response to bacterial flagellin has received considerably less attention. Here, we demonstrate that TLR5 expression by dendritic cells (DCs) allows a 1,000-fold enhancement of T-cell sensitivity to flagellin, and this enhancement did not require the expression of NLRC4 or Myd88. The effect of TLR5 on CD4 T-cell sensitivity was independent of the adjuvant effect of flagellin and TLR5 ligation did not alter the sensitivity of ovalbumin (OVA)-specific Tcells to OVA. In the spleen, the exquisite T-cell sensitivity to flagellin was regulated by CD4-CD $8 \alpha-$ DCs and was blocked by a monoclonal antibody to TLR5. In the mesenteric lymph nodes, flagellin-specific T-cell activation was regulated by a population of CD103 - CD11b + DCs. Thus, TLR5 expression by mucosal and systemic DC subsets controls the sensitivity of the adaptive immune response to flagellated pathogens.

\section{INTRODUCTION}

Flagellin subunits form the major filament of the flagella and are produced in large quantities by flagellated bacteria. ${ }^{1}$ Different leukocyte populations express surface Toll-like receptor 5 (TLR5), which can recognize extracellular flagellin and initiate an inflammatory response. ${ }^{2}$ As with many TLRs, TLR5 signaling is dependent on the recruitment of adaptor protein Myd88, but does not utilize TRIF (TIR-domain-containing adaptor-inducing interferon- $\beta)^{3}{ }^{3}$ The conserved structure of bacterial flagellins ensures that TLR5 can detect a wide array of flagellated bacteria including Listeria, Salmonella, Legionella, and Pseudomonas. ${ }^{2,4}$ Bacterial flagellins can also be recognized by the inflammasome complex and induce inflammatory responses via NAIP5 and NLRC4. ${ }^{5-8}$ Given their proinflammatory properties, flagellins can function as effective adjuvants $^{4,9}$ and are currently being tested in clinical trials. ${ }^{10,11}$

Unlike most TLR ligands, flagellins are also proteins and can be processed and presented by host major histocompatibility complex molecules and directly recognized by flagellin-specific $\mathrm{T}$ cells. ${ }^{12}$ Our laboratory and others have identified natural major histocompatibility complex class II epitopes of Salmonella flagellin, ${ }^{13-15}$ which has allowed detailed study of flagellin- specific T-cell responses in mice. ${ }^{12,16}$ Flagellin-specific CD4 T cells dominate the early immune response to intestinal Salmonella infection, ${ }^{14}$ and flagellins are also a major target antigen in murine and human inflammatory bowel disease. ${ }^{17}$ Immune reactivity to flagellins correlates with increasingly severe intestinal disease in patients with Crohn's disease. ${ }^{18,19}$ Thus, flagellins are unusual bacterial proteins that can be simultaneously recognized by multiple innate and adaptive immune receptors.

Dendritic cells (DCs) are antigen-presenting cells that are uniquely able to integrate signals from TLRs and control the activation of naive $\mathrm{T}$ cells in secondary lymphoid tissues.$^{20} \mathrm{It}$ has been thought that murine splenic DCs lack TLR5 expression as they do not produce an inflammatory response to flagellin in vitro. ${ }^{21-23}$ Indeed, a comprehensive analysis of TLR5 expression found that TLR5-expressing DCs were restricted to the intestinal lamina propria (LP). ${ }^{24}$ However, a more recent study detected a TLR5-dependent adjuvant effect in draining lymph node DCs, ${ }^{25}$ demonstrating that TLR5 can be expressed by DCs outside the intestinal LP.

Recently, we reported a requirement for TLR5 expression for induction of adaptive immune responses to flagellin after

${ }^{1}$ Center for Comparative Medicine, Department of Anatomy, Physiology, and Cell Biology, University of California Davis, Davis, California, USA and ${ }^{2}$ Laboratory of Host Defense, WPI Immunology Frontier Research Center, Osaka University, Suita, Osaka, Japan. Correspondence: SM Atif or S McSorley (smatif@ucdavis.edu or sjmcsorley@ucdavis.edu)

Received 30 July 2012; accepted 22 March 2013; published online 1 May 2013. doi:10.1038/mi.2013.25 
immunization or oral infection. ${ }^{26-29}$ Here, we have examined the role of TLR5 in DC antigen presentation of a natural flagellin epitope from Salmonella Typhimurium. We report that TLR5 is essential and NLRC4 dispensable for flagellinspecific CD4 T-cell expansion in vivo. Furthermore, expression of TLR5 allowed the host to mount a flagellin-specific immune response to very low amounts of antigen. This exquisite sensitivity to flagellin was also reproduced in vitro where TLR5 expression by DCs conferred a 1,000-fold increase in the ability to activate flagellin-specific CD4 $\mathrm{T}$ cells, an effect that was distinct from the adjuvant effect of flagellin and did not require NLRC4 or Myd88 expression. In addition, we identified $\mathrm{CD} 4-\mathrm{CD} 8 \alpha-$ DCs in the spleen as a unique subset that can regulate enhanced activation of flagellin-specific CD4 T cells. Furthermore, a corresponding population of CD103$\mathrm{CD} 11 \mathrm{~b}+\mathrm{DCs}$ isolated from the mesenteric lymph nodes (MLNs) was able to regulate T-cell sensitivity to bacterial flagellin in intestinal lymphoid tissues.

\section{RESULTS}

\section{TLR5 expression is required for the clonal expansion of} flagellin-specific $T$ cells in vivo

Given the dominance of flagellin-specific T-cell responses in infectious and inflammatory disease, ${ }^{14,17}$ we examined how
TLR5 or Myd88 expression affected the initial clonal expansion of ovalbumin (OVA)- or flagellin-specific T cells in vivo. C57BL/ 6 (wild-type) or TLR5-deficient mice were adoptively transferred with OVA-specific OT-II T cells, or flagellin-specific SM1 T cells, and immunized with a mixture of flagellin and OVA. OT-II T cells expanded similarly in wild-type, TLR5-, and Myd88deficient mice (Figure 1), demonstrating that TLR5 or Myd88 are not essential for the initial clonal expansion of OVA-specific $\mathrm{T}$ cells. In marked contrast, SM1 T cells expanded in wild-type and Myd88-deficient mice, but failed to expand in TLR5deficient mice (Figure 1). Although both SM1 and OT-II T cells expanded in Myd88-deficient mice, the level of expansion was slightly reduced compared with wild-type mice, most likely because of a reduction in the inflammatory response mediated by Myd88 (Figure 1). NLRC4 is an inflammasome component that allows intracellular recognition of flagellin, ${ }^{30}$ and has previously been implicated in the induction of flagellin-specific antibody responses. ${ }^{31}$ However, wild-type and NLRC4-deficient mice both developed robust SM1 clonal expansion 3 days after immunization of flagellin, although the level of expansion was slightly lower in NLRC4-deficient recipients (Figure 2). Together, these experiments demonstrate that TLR5 expression is required for the in vivo expansion of flagellinspecific CD4 $\mathrm{T}$ cells to flagellin immunization.
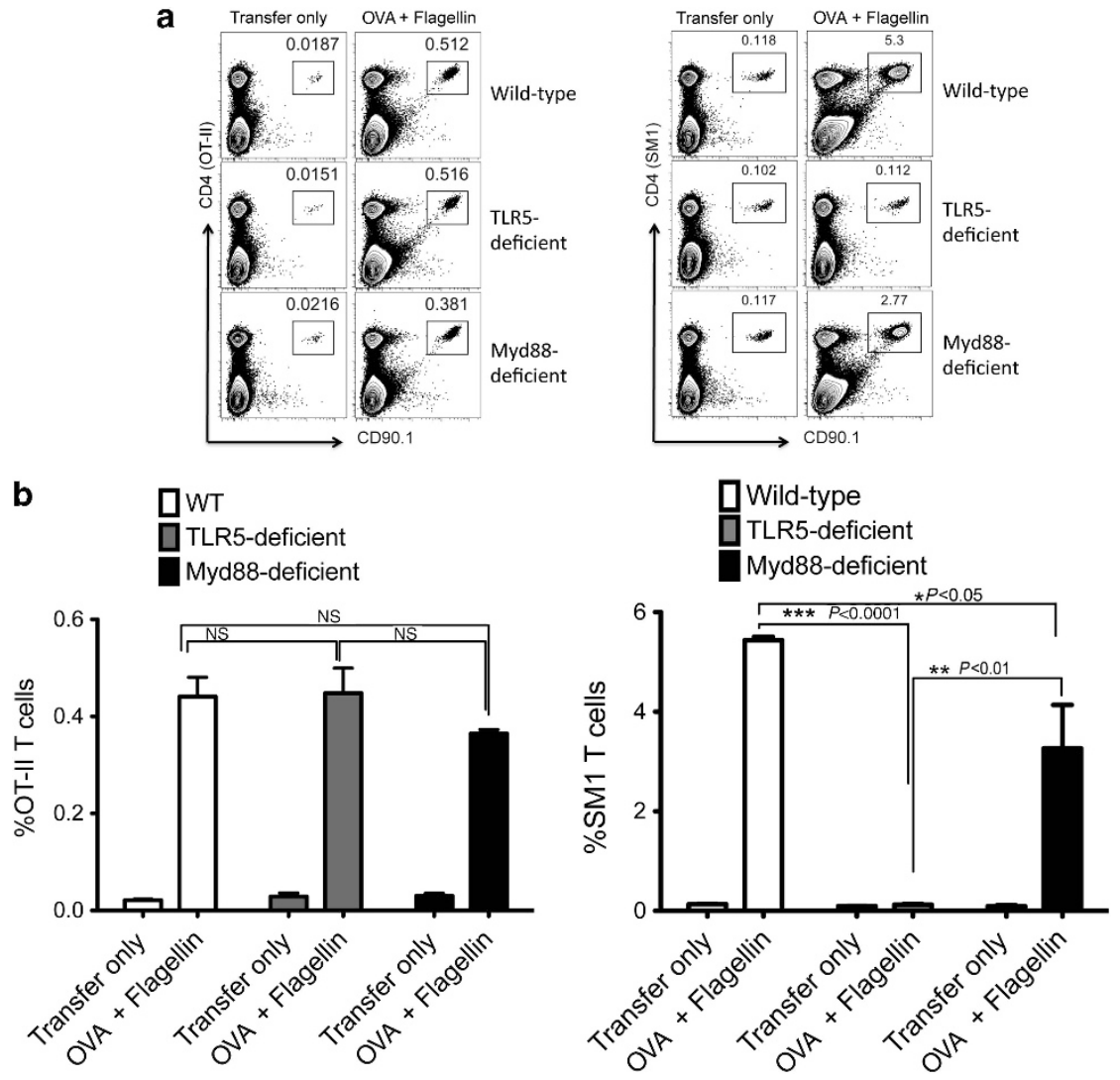

Figure 1 In vivo expansion of flagellin-specific T cells requires Toll-like receptor 5 (TLR5), but not Myd88. Wild-type, TLR5-deficient, and Myd88-deficient mice were adoptively transferred with $1 \times 10^{6}$ CD90.1 OT-II or SM1 T cell receptor (TCR) transgenic T cells and immunized with $100 \mu \mathrm{g}$ ovalbumin (OVA) plus $1 \mu \mathrm{g}$ flagellin. After 3 days, clonal expansion of OT-II and SM1 T cells was assessed in the spleen. (a) Fluorescenceactivated cell sorting (FACS) plots show CD4 and CD90.1 staining of the gating on live cells. (b) Graphs show the mean percentage \pm s.e.m. of OT-II and SM1 T cells. ${ }^{* *} P<0.0001,{ }^{* *} P<0.01$, and ${ }^{*} P<0.05$, as analyzed by two-way analysis of variance (ANOVA). NS, not significant. 

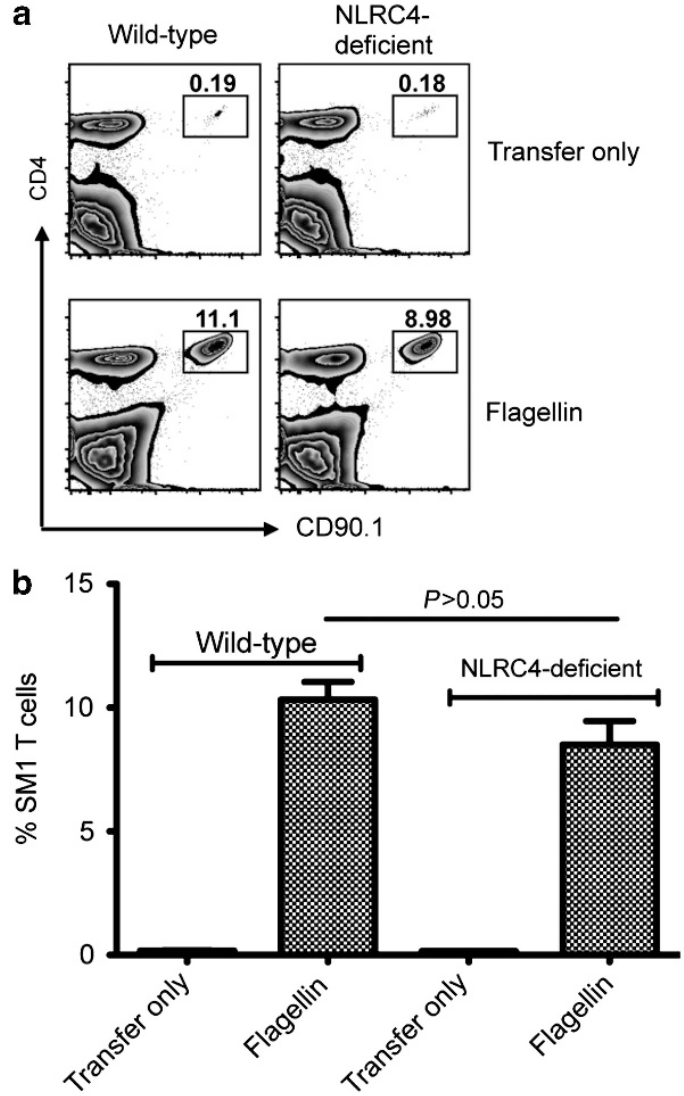

Figure 2 Robust flagellin-specific T-cell responses in NLRC4-deficient mice. Wild-type and NLRC4-deficient mice were adoptively transferred with carboxyfluorescein succinimidyl ester (CFSE)-labeled SM1 T cells and immunized intravenously the following day with $1 \mu \mathrm{g}$ of flagellin and $10 \mu \mathrm{g}$ of lipopolysaccharide (LPS). (a) Fluorescence-activated cell sorting (FACS) plots show expansion of SM1 T cells (CD4 and CD90.1 positive) in the spleen of wild-type and NLRC4-deficient mice 3 days after immunization. (b) Bar plots show the percentage of SM1 T cells in the spleen of wild-type and NLRC4-deficient mice 3 days after immunization. There is no significant difference between immunized groups, as evaluated by unpaired $t$-test $(P<0.05)$.

\section{TLR5 regulates adaptive immune sensitivity to bacterial flagellin}

The experiments above suggested that TLR5 controls the host adaptive immune response to bacterial flagellin. In order to determine the in vivo threshold for antigen-specific T-cell activation, we immunized mice with varying concentrations of OVA and flagellin and examined the clonal expansion and carboxyfluorescein succinimidyl ester (CFSE) dye dilution of OT-II and SM1 T cells. In wild-type mice, OT-II T cells were activated to undergo CFSE dye dilution, expansion, and increased surface expression of CD11a, following immunization with 10-100 $\mu \mathrm{g}$ OVA (Figure 3a,d). Importantly, OT-II T cells did not respond to immunization with $1 \mu \mathrm{g}$ OVA, remained CD11a ${ }^{\text {low }}$, and did not dilute CFSE (Figure 3a,d). In marked contrast, SM1 T cells diluted CFSE, clonally expanded, and increased surface expression of CD11a after immunization with as little as $0.0001 \mu \mathrm{g}(100 \mathrm{pg})$ of flagellin (Figure $3 \mathbf{b}, \mathbf{e})$. This exquisite sensitivity to low-dose flagellin immunization was absent in mice lacking TLR5 (Figure 3c,f). Indeed, SM1 T cells in TLR5-deficient mice were only able to respond to flagellin when doses of $1-100 \mu \mathrm{g}$ were used (Figure 3c,f). This effect was distinct from the intrinsic adjuvant effect of flagellin as the expansion of OT-II T cells was unaffected by TLR5 deficiency, even when flagellin was used as an adjuvant, and T-cell expansion was also detected in Myd88deficient mice (Figure 1). Thus, host expression of an innate receptor for flagellin (TLR5) allows for a 1,000-fold enhancement in the sensitivity of the adaptive immune response to this same antigen.

\section{DC expression of TLR5 controls T-cell sensitivity to flagellin}

Lymph node and splenic DCs are not thought to express TLR5 as these populations do not generate an inflammatory response to flagellins in vitro. ${ }^{21-23}$ We examined whether TLR5 expression by splenic DCs was responsible for the sensitivity of flagellin-specific T-cell activation. CD11c + DCs from the spleens of wild-type and TLR5-deficient mice were enriched by magnetic selection (Supplementary Figure S1A online). CD11c + DCs were incubated with OT-II or SM1 T cells in the presence or absence of OVA or flagellin, and T-cell activation was subsequently examined. At $16 \mathrm{~h}$ after incubation with $10-100 \mu \mathrm{g} \mathrm{ml}^{-1}$ of OVA, OT-II T cells displayed increased expression of CD69, whereas lower doses failed to activate OVA-specific T cells (Figure 4a, solid line). In a similar manner, SM1 T cells incubated with TLR5-deficient DCs were activated to express CD69 when a range of $1-100 \mu \mathrm{g} \mathrm{ml}^{-1}$ of flagellin was added to cultures (Figure $\mathbf{4 b}$, dotted line). In marked contrast, DCs from wild-type mice were capable of activating SM1 T cells to express CD69 even when as little as $1 \mathrm{ng} \mathrm{ml}^{-1}$ of flagellin was used (Figure $\mathbf{4 b}$, solid line). This difference in antigen-presenting capacity at low doses was absent when flagellin peptide was used as a stimulus rather than whole flagellin protein (Supplementary Figure S2 online), indicating that the enhanced sensitivity to flagellin in wild-type DCs is a consequence of TLR5 expression rather than intrinsic differences in the capacity to present antigen. This TLR5dependent effect was also distinct from the adjuvant effect of flagellin in vitro, as the addition of flagellin in vitro did not improve the sensitivity of the OT-II responses to OVA (Figure 4c). Furthermore, activation of OT-II cells was largely unaffected by TLR5 deficiency, even when flagellin was added to cultures (Figure 4d).

Next, we examined the expression of multiple markers of CD4 T-cell activation following stimulation of flagellin-specific $\mathrm{T}$ cells with low-dose $\left(1 \mathrm{ng} \mathrm{ml}^{-1}\right)$ flagellin. Wild-type DCs induced SM1 T cells to increase the expression of CD25 and CD69 at $16 \mathrm{~h}$ after adding flagellin, whereas TLR5-deficient DCs did not (Figure 5a). Similarly, at $48 \mathrm{~h}$, wild-type DCs induced SM1 T cells to increase expression of CD44 and reduce expression of CD62L, whereas TLR5-deficient DCs did not (Figure 5a). Wild-type DCs also induced interleukin-2 (IL-2) production from naive SM1 T cells at much lower flagellin concentrations than TLR5-deficient DCs (Figure 5b). 
a

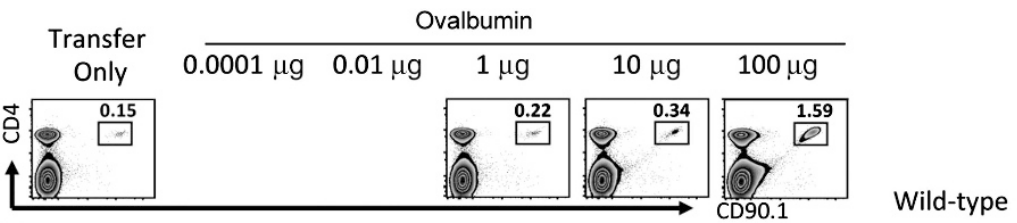

b
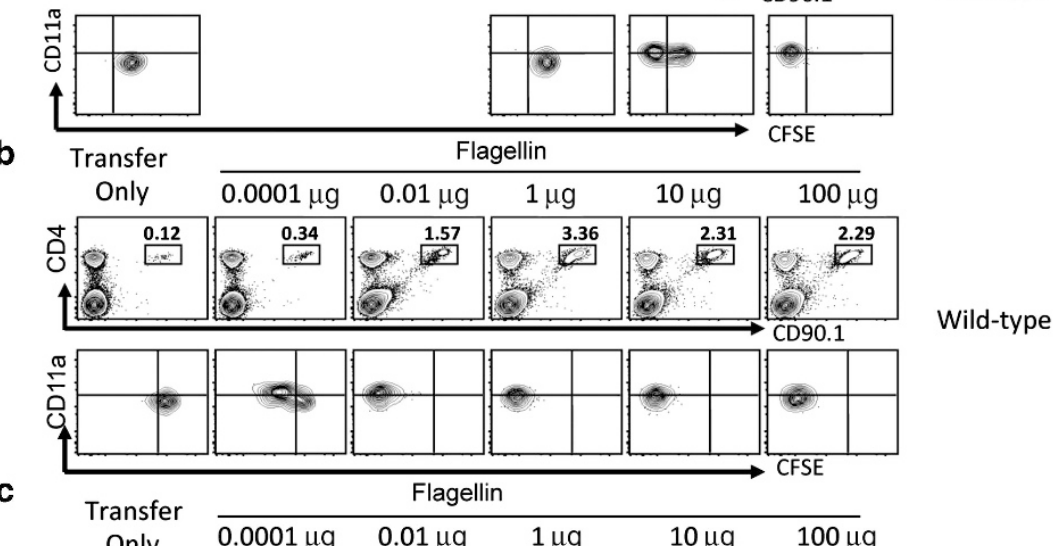

\section{Wild-type}
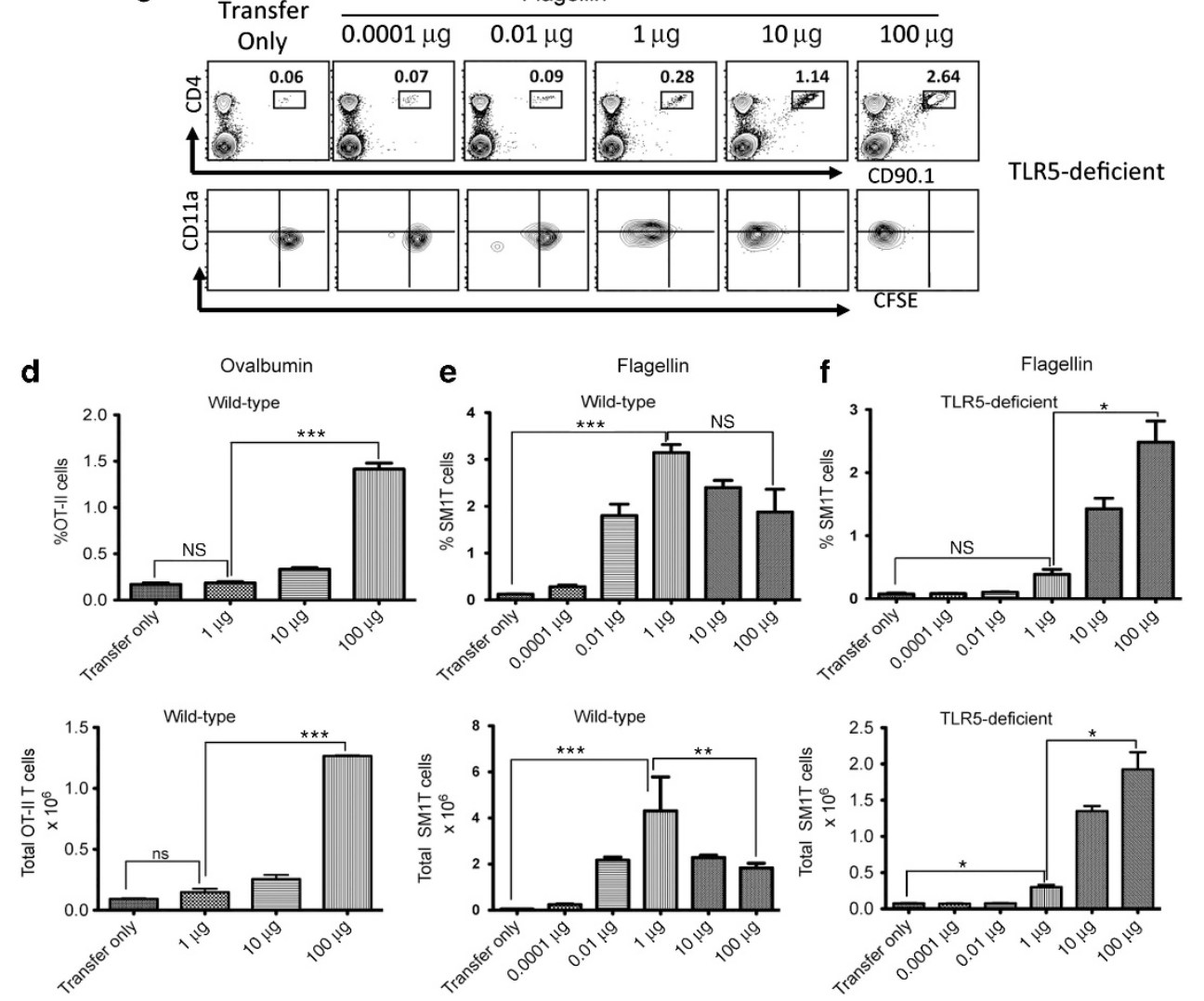

Figure 3 Toll-like receptor 5 (TLR5) enhances T-cell activation to low doses of flagellin. $1 \times 10^{6}$ carboxyfluorescein succinimidyl ester (CFSE)-labeled $\mathrm{T}$ cell receptor (TCR) transgenic T cells were adoptively transferred into wild-type and TLR5-deficient mice and the following day were immunized with different doses of ovalbumin (OVA; $1-100 \mu \mathrm{g}$ ) or flagellin (100 pg to $100 \mu \mathrm{g})$ plus $10 \mu \mathrm{g}$ of lipopolysaccharide (LPS). After 3 days, clonal expansion of (a) OT-II or (b and c) SM1 T cells was examined in the spleen. Upper plots show staining of CD4 and CD90.1 to identify transgenic T cells and lower plots show CD11a and carboxyfluorescein succinimidyl ester (CFSE) staining after gating on OT-II and SM1 T cells. Each plot is a representative of three mice per group and two independent experiments. (d) Bar plots showing the percentage and total number of OT-II T cells in the spleen of OVA $(1-100 \mu \mathrm{g})$ immunized animals. (e, f) Plots show the percentage and total number ( \pm s.e.m.) of SM1 T cells in the spleen of (e) wild-type and (f) TLR5-deficient mice immunized with flagellin. Graphs combine the results of two independent experiments with three mice per group. Statistical significance was examined using unpaired $t$-test between the groups immunized with OVA $(100 \mu \mathrm{g})$ or flagellin $(1 \mu \mathrm{g})$ and transfer only. ${ }^{* * *} P<0.0001,{ }^{* *} P<0.01$, and ${ }^{*} P<0.05$, as analyzed by unpaired $t$-test.

In contrast, TLR5-deficient DCs were able to induce an equivalent response if flagellin peptide (427-441) was used to stimulate SM1 cells (Figure 5b). As Myd88 expression is required for all previously reported functions of TLR5, ${ }^{4}$ we examined the activation of SM1 T cells using Myd88-deficient DCs. Myd88-deficient DCs remained fully capable of activating 


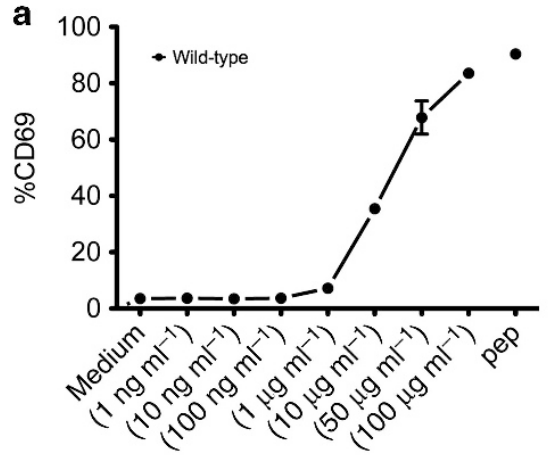

C

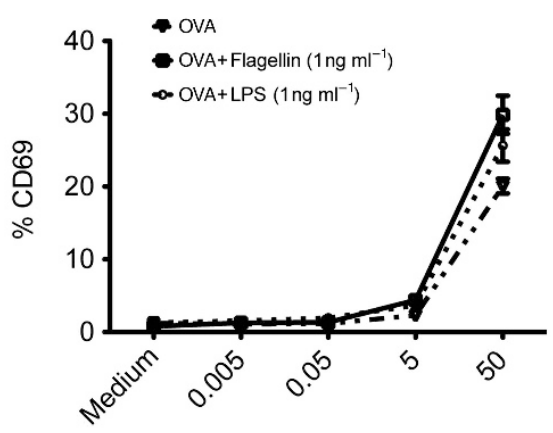

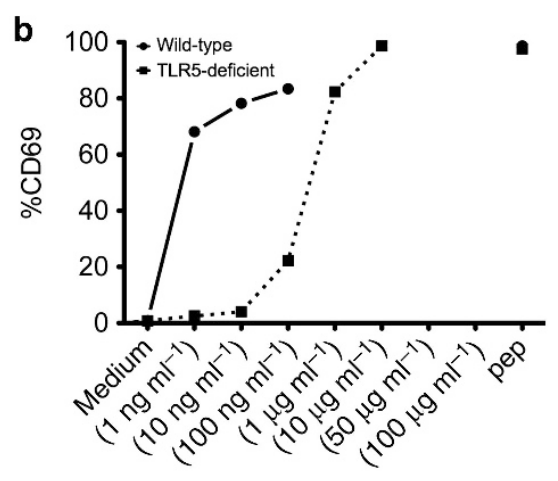

d

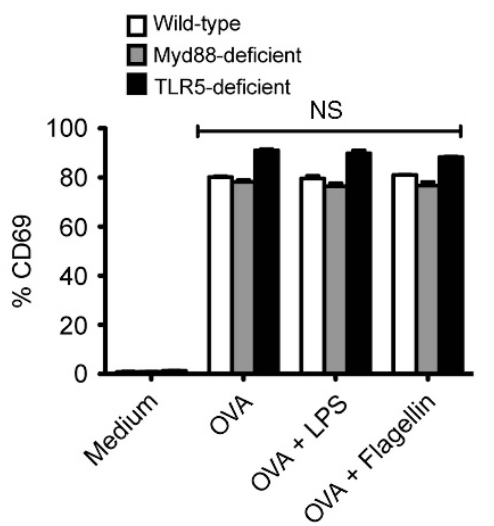

Figure 4 Toll-like receptor 5 (TLR5) expression by CD11c + dendritic cells (DCs) controls the sensitivity of flagellin-specific T-cell activation. DCs isolated from C57BL/6 (wild-type) and TLR5-deficient mice were cultured with T cell receptor (TCR) transgenic (SM1 or OT-II) T cells and various concentrations of antigen (flagellin or ovalbumin (OVA)). (a) Graph shows increased expression of CD69 on gated (CD4 + CD90.1 + ) OT-II T cells after $16 \mathrm{~h}$ of incubation with wild-type DCs and OVA (1 $\mathrm{ng} \mathrm{ml}^{-1}$ to $\left.100 \mu \mathrm{g} \mathrm{m}^{-1}\right)$. (b) Graph shows increased expression of CD69 on (CD4 + CD90.1 +) SM1 T cells after 16h of incubation in the presence of wild-type (solid line) or TLR5-deficient DCs (dotted line) with flagellin $\left(1 \mathrm{ng} \mathrm{ml}^{-1}\right.$ to $\left.10 \mu \mathrm{g} \mathrm{ml}^{-1}\right)$ or flagellin peptide $(6 \mu \mathrm{M})$. (c) DCs were cultured with TCR transgenic OT-II T cells and various concentrations of OVA ( $\mu \mathrm{g} \mathrm{ml}^{-1}$ ), OVA plus flagellin, or OVA plus lipopolysaccharide (LPS). Graph shows increased expression of CD69 on gated (CD4 + CD90.1 + ) OT-II T cells after $16 \mathrm{~h}$ of incubation with wild-type DCs. (d) DCs isolated from wild-type, TLR5-deficient, or Myd88-deficient mice were cultured with OT-II T cells and OVA $\left(100 \mu \mathrm{g} \mathrm{ml}^{-1}\right)$ or OVA plus flagellin $\left(1 \mathrm{ng} \mathrm{ml}^{-1}\right)$, or OVA plus LPS $\left(1 \mathrm{ng} \mathrm{ml}^{-1}\right)$. Graph shows increased expression of CD69 on (CD4 + CD90.1 +) OT-II T cells after $16 \mathrm{~h}$ of incubation with wild-type, TLR5-deficient, or Myd88-deficient DCs.
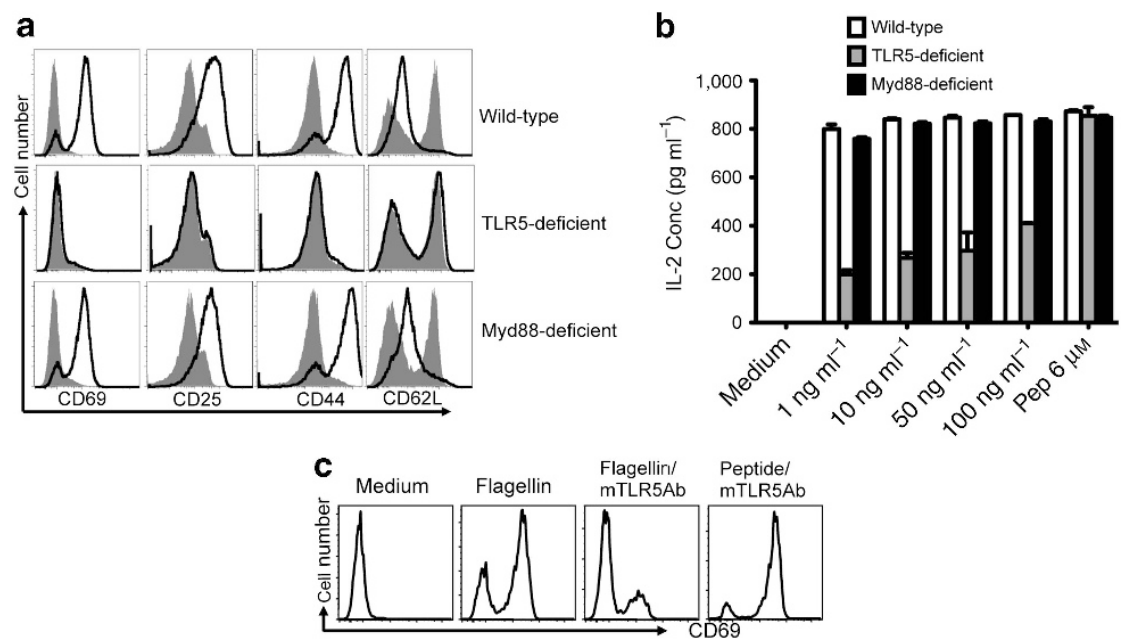

Figure 5 Dendritic cell (DC) expression of Toll-like receptor 5 (TLR5), but not Myd88, is required to activate flagellin-specific T cells. DCs from the spleen of wild-type, TLR5-deficient, and Myd88-deficient mice were incubated with flagellin and SM1 T cells to examine T-cell activation. $(\mathbf{a}, \mathbf{b}) 1 \times 10^{5}$ DCs were cultured with $1 \times 10^{5} \mathrm{SM} 1 \mathrm{~T}$ cells for $16 \mathrm{~h}$ (CD69 and CD25) or $48 \mathrm{~h}$ (CD44 and CD62L) in the presence of $10 \mathrm{ng} \mathrm{ml}^{-1}$ of flagellin (solid line) or medium alone (shaded area). (a) Plots show cell surface expression of CD69, CD25, CD44, or CD62L after gating on (CD4 + CD90.1 +) SM1 T cells.

(b) Production of interleukin-2 (IL-2) in culture supernatants was assessed by enzyme-linked immunosorbent assay (ELISA) $48 \mathrm{~h}$ after incubation with different concentrations (Conc) of flagellin or medium alone. Graph shows mean IL-2 \pm s.e.m. in tissue culture replicates after stimulation.

(c) Prior treatment of DCs with a monoclonal anti-TLR5 antibody (mTLR5Ab; $10 \mu \mathrm{g} \mathrm{ml}^{-1}$ ) for 60 min inhibited flagellin-specific activation of SM1 T cells. Plots show CD69 surface staining after gating on CD4 + CD90.1 SM1 T cells. All data are representative of three independent experiments. 
SM1 T cells to modulate expression of activation markers and secrete IL-2 (Figure 5a,b). Thus, the sensitivity of flagellinspecific T cells is controlled by DC expression of TLR5, but does not require the expression of Myd88.

Next, we examined whether inhibition of flagellin binding to TLR5 could interfere with the ability of DCs to activate flagellin-specific T cells. Wild-type CD11c + DCs isolated from the spleen were incubated with SM1 T cells, flagellin, and a blocking TLR5 monoclonal antibody. Inhibition of TLR5 binding eliminated SM1 T-cell activation (Figure 5c), whereas this same antibody had no effect on the T-cell response to processed flagellin peptide 427-441 (Figure 5c). Thus, physical interaction of flagellin with TLR5 on DCs is a prerequisite for TLR5 to enhance flagellin-specific T-cell responses.

\section{Splenic CD4 - CD8 $\alpha$ - myeloid DCs control T-cell responses to flagellin}

CD11c + splenic DCs are a heterogeneous population and several different subsets have been defined based on the expression of $\mathrm{CD} 8 \alpha, \mathrm{CD} 1 \mathrm{~b}$, and $\mathrm{CD} 4 .^{32,33} \mathrm{We}$ examined whether these different splenic DC subsets were differentially able to activate flagellin-specific $\mathrm{T}$ cells in vitro. Initially, we isolated $\mathrm{CD} 11 c+\mathrm{CD} 8 \alpha+$ and $\mathrm{CD} 11 c+\mathrm{CD} 8 \alpha-\mathrm{DCs}$ and found that only myeloid (CD11c + CD $8 \alpha-$ ) DCs were capable of activating SM1 T cells to express CD69 and CD25 using flagellin as an antigen (Figure 6a). Examining this myeloid DC population further, we found that only CD11c $+\mathrm{CD} 8 \alpha-\mathrm{DCs}$ that lack CD4 expression were able to efficiently activate SM1 T cells in vitro, whereas CD4-expressing DCs did not (Figure 6b). Thus, the ability to sensitively activate flagellin-specific T cells is regulated by a minor population of splenic DCs that lack expression of both CD 4 and CD $8 \alpha$.

\section{MLN CD103 - CD11b + DCs regulate T-cell responses to flagellin}

Flagellin-specific T-cell responses are often induced in the intestine and thus host immune sensitivity to flagellin is likely to be regulated by intestinal DC subsets. ${ }^{26,34,35}$ We therefore compared the ability of DCs from spleen, peripheral lymph nodes, and MLNs of wild-type and TLR5-deficient mice to activate SM1 T cells in vitro. CD11c + DCs from each of these locations were able to activate flagellin-specific $\mathrm{T}$ cells in vitro to express CD69, and the expression of TLR5 was required in each case (Figure 7a-c). In contrast, wild-type and TLR5-deficient DCs were equivalently able to activate OT-II cells incubated in the presence of OVA (Supplementary Figure S3 online). In addition, flagellin-specific T-cell activation by wild-type DCs was blocked by the addition of an anti-TLR5 monoclonal antibody (Figure 7d). In contrast, CD11c + DCs from the Peyer's Patch or LP were poorly able to activate flagellinspecific T cells (Supplementary Figure S4 online), although the CD11c enrichment efficiency was somewhat lower for both these tissues.

Next, we enriched CD103 + and CD103 - MLN DCs and examined whether these populations were differentially able to present flagellin (Supplementary Figure S1 online). Although CD103 + DCs were relatively poor at activating SM1 T cells,
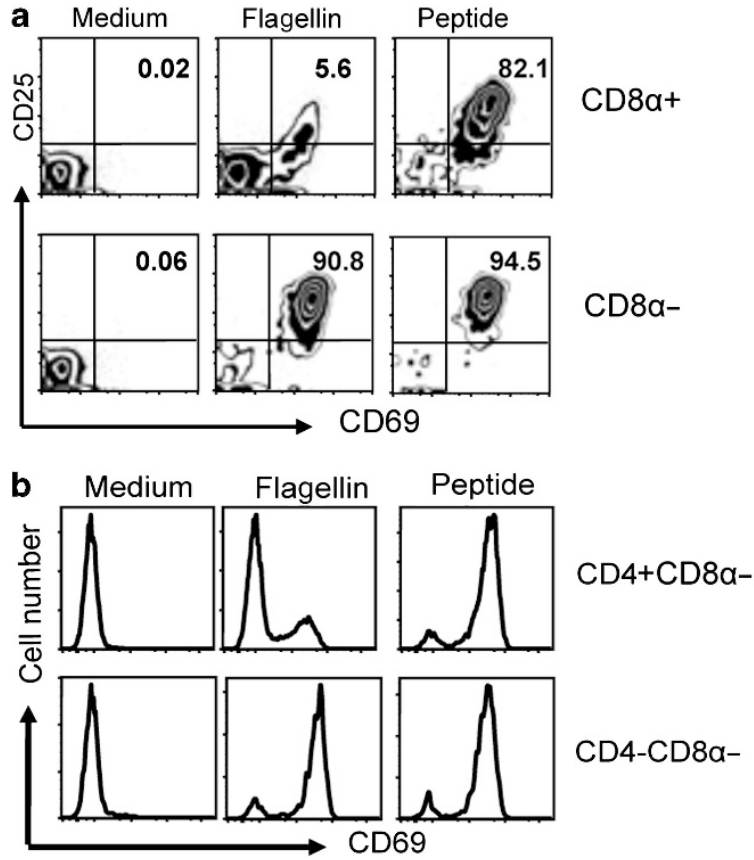

Figure 6 Splenic dendritic cell (DC) subsets have differential ability to activate flagellin-specific T cells. CD $8 \alpha+, \mathrm{CD} 8 \alpha-, \mathrm{CD} 4+\mathrm{CD} 8 \alpha-$, and CD $4-C D 8 \alpha$ - splenic CD11c + DC subsets were purified using DC enrichment kits and were analyzed for their ability to activate flagellinspecific T cells in vitro. DC subsets were incubated with SM1 T cells in a 1:1 ratio $\left(1 \times 10^{5}\right.$ cells per well) in the presence of $10 \mathrm{ng} \mathrm{ml}^{-1}$ of flagellin or $6 \mu \mathrm{m}$ of flagellin peptide (427-441). (a) Upper and lower panel show the activation of SM1 T cells in the presence of CD11c + CD $8 \alpha+$ lymphoid or CD11c + CD8 $\alpha$-myeloid DCs as evaluated by CD69 and CD25 expression on gated SM1 T cells at $16 \mathrm{~h}$ after incubation. (b) CD $4+\mathrm{CD} 8 \alpha-$ and CD4CD $8 \alpha$ - myeloid DC subsets $1 \times 10^{5}$ were co-cultured with $1 \times 10^{5}$ SM1 T cells for $16 \mathrm{~h}$ in the presence of flagellin and T-cell activation analyzed by examining CD69 expression on gated SM1 T cells. Data are representative of two independent experiments completed in triplicate.

there was a small but significant increase in T- cell activation when CD103 - DCs were used (Figure 8a). Similar to splenic DCs, when MLN DCs were enriched based on expression of CD11b, there was a significant enhancement in the ability to present flagellin to SM1 T cells (Figure 8b). Furthermore, the ability of MLN CD11b + DCs to present flagellin was blocked by the addition of a monoclonal antibody against TLR5 (Figure 8c). The difference in the ability of CD11b + DCs to activate flagellin-specific $\mathrm{T}$ cells also correlated with increased TLR5 expression by this subset, as examined by reverse transcriptase-PCR analysis (Supplementary Figure S5 online). Together, these data suggest that $\mathrm{CD} 103$ - and $\mathrm{CD} 11 \mathrm{~b}+$ expression define a MLN DC subset with enhanced ability to initiate flagellin-specific T-cell responses to low doses of flagellin.

\section{DISCUSSION}

Immunologists have historically devoted a significant amount of attention to examining flagellin-specific immune responses due to the immunogenicity of this protein. ${ }^{36-39}$ For example, it was noted more than 40 years ago that immune response in rats can be induced with as little as $10 \mathrm{ng}$ of flagellin. ${ }^{36,38}$ Our data 
a
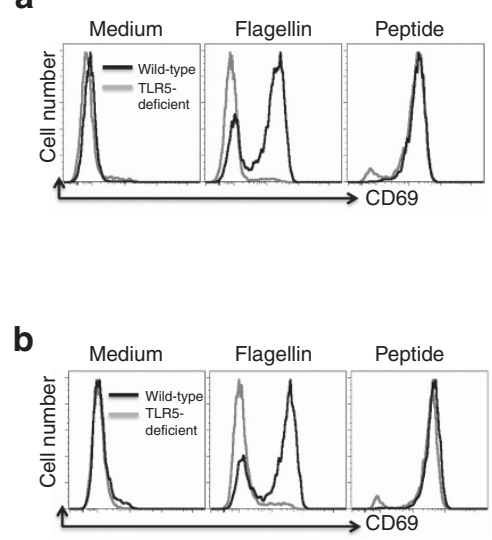

C

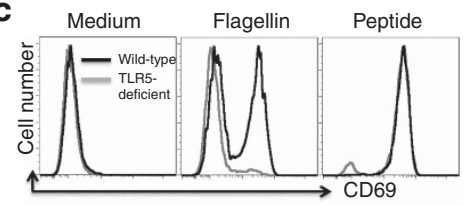

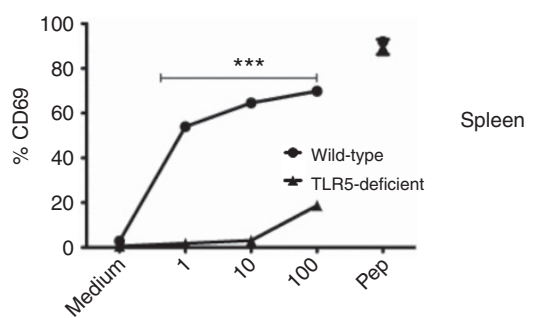

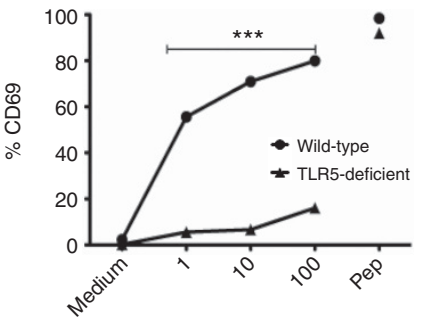

Peripheral

Lymph nodes

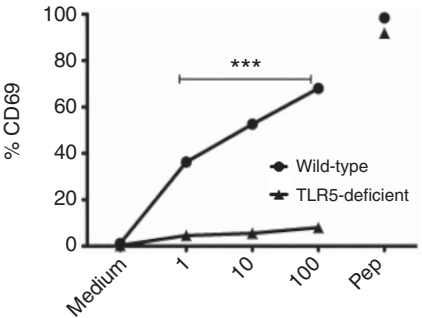

Mesenteric

Lymph nodes

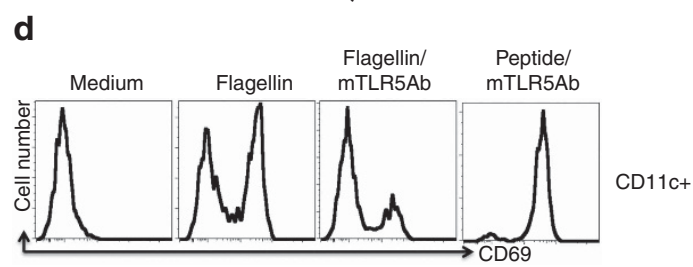

Figure 7 Toll-like receptor 5 (TLR5) is required for splenic and mucosal dendritic cells (DCs) to activate flagellin-specific T cells. CD11c + DCs enriched from (a) spleen, (b) peripheral lymph nodes, or (c) mesenteric lymph nodes of wild-type and TLR5-deficient mice. Fluorescence-activated cell sorting (FACS) plots (left) show activation of SM1 T cells after $16 \mathrm{~h}$ of culture with wild-type DCs (black) or TLR5-deficient DCs (gray) in the presence or absence of $10 \mathrm{ng} \mathrm{ml}^{-1}$ of flagellin or flagellin peptide $(6 \mu \mathrm{M})$. T-cell activation was analyzed by the expression of CD69 on gated SM1 T cells (CD4 + CD90.1 +). Graphs (right) show increased expression of CD69 on (CD4 + CD90.1 +) SM1 T cells after 16 h of incubation in the presence of wild-type (•) or TLR5deficient DCs $(\mathbf{\Delta})$ with flagellin $(1-100 \mathrm{ng}) \mathrm{ml}^{-1}$ or flagellin peptide $(6 \mu \mathrm{m})$. Each data point is representative of mean of two independent experiments done in triplicate; ${ }^{* \star} P<0.0001$, as analyzed by two-way analysis of variance (ANOVA). (d) CD11c + DCs from mesenteric lymph nodes were incubated with monoclonal TLR5 antibody (mTLR5Ab; $10 \mu \mathrm{g} \mathrm{ml}^{-1}$ ) for $60 \mathrm{~min}$ and then cultured in a 1:1 ratio with SM1 T cells in the presence of flagellin (1 $\mathrm{ng} \mathrm{ml}^{-1}$ ) and flagellin peptide $(6 \mu \mathrm{M})$. After $16 \mathrm{~h}$, CD69 expression was measured on T cells by flow cytometry. Data are representative of two independent experiments.

demonstrate that TLR5 expression largely accounts for the exquisite sensitivity of a mammalian host to respond to bacterial flagellin. This finding is important as it also provides a plausible explanation for the underlying dominance of flagellin-specific immune responses in infectious and inflammatory diseases of the intestine, perhaps especially so when overall antigen concentrations are very low, such as during the early stages of bacterial infection. However, it is also important to note that TLR5-deficient mice retain the ability to respond to concentrations of flagellin in the microgram range and this is similar to the threshold for OVA-specific responses. Thus, expression of TLR 5 simply confers a unique sensitivity to low doses of flagellin in wild-type mice. Further studies will be required to determine whether the expression of TLR5 is required for directing flagellin-specific adaptive immunity during infection or inflammatory bowel disease.

Bacterial flagellin can also be recognized by NLRC4 (earlier known as ICE like protease activating factor (IPAF)) and NAIP5 in the cytosol. ${ }^{5-8}$ Recently, NLRC4 was reported to be essential for humoral responses to flagellin. ${ }^{31}$ However, our data demonstrate that NLRC4 plays at most a minor role in directing flagellin-specific T-cell activation, as the expansion and activation of SM1 T cells was similar in mice expressing or lacking NLRC4. Given the cytosolic location of inflammasome detection, it remains possible that NLRC4 plays a more important role in directing the CD8 T-cell responses; however, reagents to study flagellin-specific CD8 $\mathrm{T}$ cells are not yet available. 

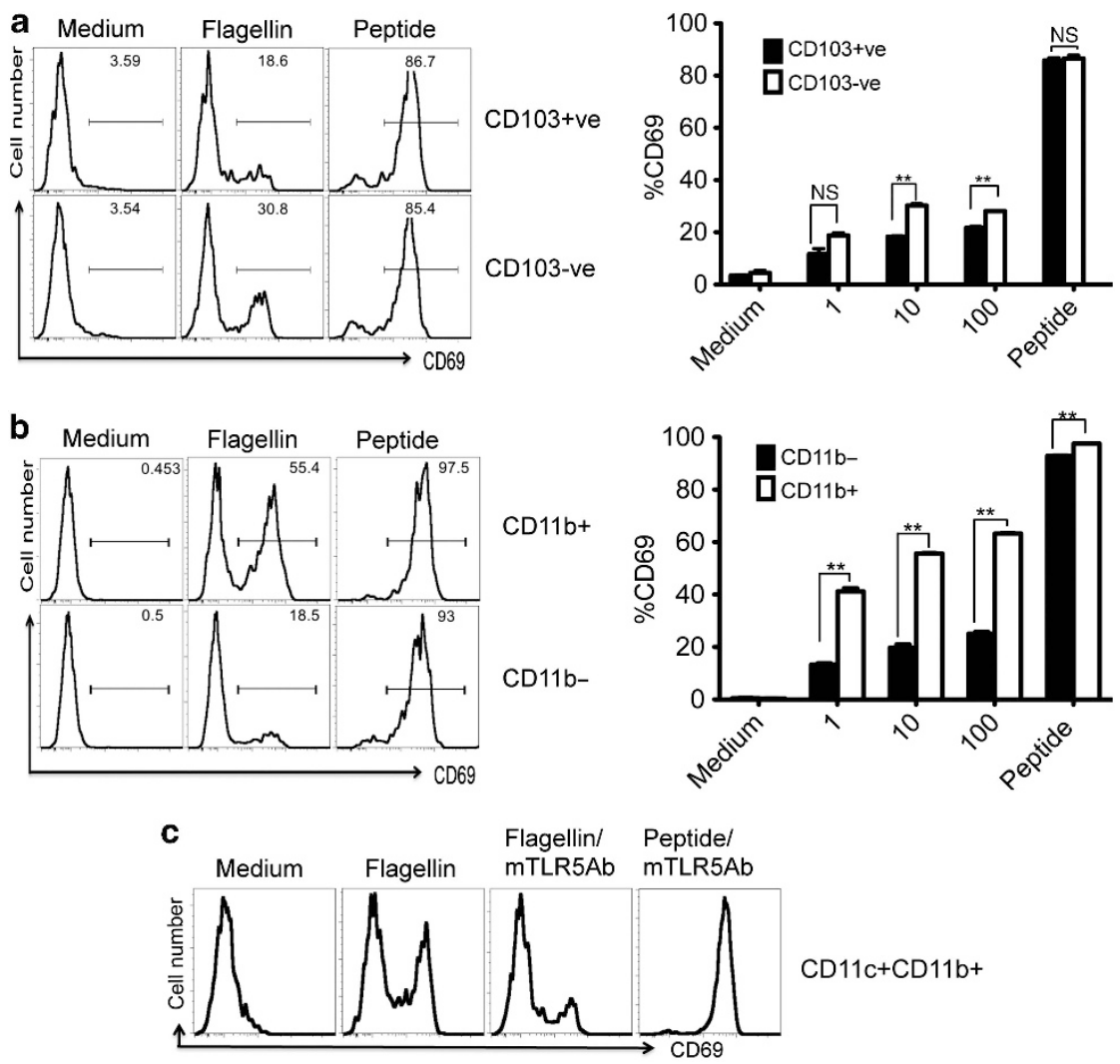

Figure 8 Mesenteric lymph node (MLN) dendritic cell (DC) subsets have differential ability to activate flagellin-specific T cells. (a) CD103-positive or -negative myeloid DCs from MLNs were enriched from CD11c + DCs using magnetic beads and cultured with SM1 T cells for $16 \mathrm{~h}$ in the presence of flagellin $\left(10 \mathrm{ng} \mathrm{ml}^{-1}\right)$ or flagellin peptide $(6 \mu \mathrm{m})$. Fluorescence-activated cell sorting (FACS) plots (left) show CD69 expression on gated SM1 T cells. Bar graphs (right) show dose-dependent activation of SM1 T cells cultured with CD103-positive or -negative fractions. Each bar shows the mean percentage of CD69 + cells after gating on SM1 T cells. (b) FACS plots show activation of SM1 T cells cultured for 16 h with CD11b-positive or -negative DC fraction in the presence or absence of flagellin $\left(10 \mathrm{ng} \mathrm{ml}^{-1}\right)$ or flagellin peptide $(6 \mu \mathrm{M})$. Bar graphs (right) show dose-dependent activation of SM1 T cells cultured with CD11b + or CD11b - MLN DCs. Each bar shows the mean percentage of CD69+ cells after gating on SM1 T cells. (c) CD11b + DCs pretreated with monoclonal anti-Toll-like receptor 5 antibody (mTLR5Ab) were cultured with SM1 T cells and T-cell activation was examined $16 \mathrm{~h}$ later. ${ }^{* \star} P<0.01$; NS, $P>0.05$ as analyzed by unpaired $t$-test.

The spleen contains multiple DC subsets including CD4$\mathrm{CD} 8 \alpha+, \mathrm{CD} 4+\mathrm{CD} 8 \alpha-$, and $\mathrm{CD} 4-\mathrm{CD} 8 \alpha-$ populations. ${ }^{33}$ $\mathrm{CD} 8 \alpha+$ lymphoid DCs are prominent in the white pulp whereas CD $8 \alpha$ - myeloid DCs localize to the marginal zone and bridging channels. ${ }^{40}$ Here, we show that myeloid CD4 DCs are also functionally specialized to activate flagellinspecific $T$ cells at very low antigen concentrations. Similarly, the MLN contains multiple resident and migratory DC subsets and our data pinpoint CD11c $+\mathrm{CD} 103-\mathrm{CD} 11 \mathrm{~b}+\mathrm{DCs}$ as a subset that is specifically capable of activating flagellin-specific $\mathrm{T}$-cell response at very low antigen concentrations. This subset is likely a blood-derived resident DC population that is found in all secondary lymphoid tissues, although further studies will be required to demonstrate this. The enhanced capacity to activate flagellin-specific $\mathrm{T}$ cells required the expression of TLR5 and was blocked by anti-TLR5. It seems likely therefore that TLR5 allows preferential delivery of flagellin to the major histocompatibility complex class II loading compartment, although this remains to be demonstrated in vitro or in vivo. It is important to note that the ability of these DC subsets to present flagellin to T cells is TLR5 dependent but independent of
Myd88 and can be detected in splenic DCs that show low inflammatory responses to flagellin in vitro. Thus, this functionality is distinct from the inflammatory activity of flagellin mediated by TLR5 and could therefore require a coreceptor or unique signaling components. Indeed, a population of $\mathrm{CD} 103+$ DCs in the LP have been noted to display inflammatory activity in response to flagellin, ${ }^{41}$ but we detected low responses from LP DCs in T-cell stimulation assays. These data suggest that different intestinal DC subsets may use TLR5 differentially to induce inflammatory responses in intestinal tissue or present antigen to CD4 T cells in secondary lymphoid tissues.

It is not yet clear whether the increased sensitivity to antigen conferred by TLR 5 expression is typical of other TLRs. TLR11 also recognizes a protein ligand, profilin, and indeed, profilinspecific CD4 $\mathrm{T}$ cells are induced in a TLR11-dependent manner. ${ }^{42}$ It is possible that any protein ligand that is internalized via surface TLRs benefits from enhanced delivery to the class II processing pathway and therefore the mechanism of this enhanced delivery will be of interest to vaccine design. In conclusion, our study demonstrates the importance of TLR5 in 
DC antigen presentation by decreasing the threshold at which flagellin-specific $\mathrm{T}$ cells are activated and also suggests that TLR5 expression by distinct populations of splenic and intestinal dendritic cells are responsible for focusing host responses onto this antigen in infectious and inflammatory disease states. Given these findings, it is possible that this novel function of TLR5 can be utilized to improve the immunogenicity of enteric vaccines or prevent flagellin-specific responses in inflammatory disease.

\section{METHODS}

Mice and reagents. C57BL/6 and B6.SJL-PtprcaPep3b/BoyJ (CD45.1 congenic) mice (6-8 weeks old) were purchased from The Jackson Laboratory (Bar Harbor, ME) and NCI (Frederick, MD). TLR5deficient and NLRC4-deficient mice ${ }^{24,43}$ were bred from stock originally provided by Dr A. Gewirtz (Georgia State University, Atlanta, GA). Myd88-deficient mice ${ }^{44}$ were provided by Dr S. Way (University of Minnesota Medical School, Minneapolis, MN). Rag-deficient, CD90.1 congenic, flagellin-specific SM1 T cell receptor transgenic mice have been described previously. ${ }^{26,27}$ Rag-deficient, OT-II T cell receptor transgenic mice ${ }^{45}$ were backcrossed to a Rag-deficient CD90.1 congenic background in our laboratory. The flagellin epitope recognized by SM1 T cells (flagellin ${ }_{427-441}$ ) has been reported, ${ }^{14}$ and this peptide was purchased from Invitrogen (Carlsbad, CA).

Bacterial strains and flagellin production. Lipopolysaccharidedeficient Salmonella serovar Typhimurium X4700, provided by Dr R Curtiss (Arizona State University, Tempe, AZ), was used to purify flagellin using a modified acid-shock protocol. Overnight, LB broth cultures were centrifuged, washed, and resuspended in phosphatebuffered saline, before acid treatment to liberate flagellin. Monomeric flagellin was prepared by depolymerizing dialyzed samples at $70{ }^{\circ} \mathrm{C}$ for $1 \mathrm{~h}$ and passed through endotoxin removal columns (Pierce Biotechnology, Rockford, IL). Flagellin purity was determined using silver stained sodium dodecyl sulfate gels and endotoxin detection kits (Sigma, St Louis, MO). Our flagellin preparations have identical biological activity to recombinant flagellin produced by eukaryotic cells. $^{22}$

Adoptive transfer and immunization. Spleen, inguinal, brachial, cervical, and MLNs were harvested from SM1 or OT-II mice and red blood cells were lysed using ACK lysis buffer (Lonza, Walkersville, MD) before labeling with CFSE. ${ }^{46}$ The percentage of transgenic T cells was determined and 800,000-1 $\times 10^{6}$ cells transferred intravenously into mice. The following day, mice were injected intravenously with various doses of flagellin or OVA.

DC enrichment and in vitro stimulation. Spleens, peripheral lymph nodes, and MLNs were digested using collagenase D (Roche Diagnostics, Indianapolis, IN) ${ }^{22}$ and DCs enriched to greater than $85-95 \%$ purity using CD11c microbeads (Miltenyi Biotech, Auburn, CA) (Supplementary Figure S1 online). DC subsets were enriched by positive or negative selection using antibodies specific for CD11c, CD11b, CD103, or CD8 $\alpha$ (Miltenyi Biotech). Further separation of splenic myeloid CD8 $\alpha$ - DCs was accomplished using a CD4 isolation kit (Miltenyi Biotech). CD11b + DCs were enriched using CD11b + isolation kit (Milteyni Biotech; Supplementary Figure S1 online). CD103 + DCs were isolated from negatively enriched CD11c + DCs using CD103-specific antibody and streptavidin beads (Milteyni Biotech). The unbound CD103 - fraction was used to purify CD11b + DCs. DC subsets $\left(1 \times 10^{5}\right.$ cells per well $)$ were incubated 1:1 with SM1 or OT-II T cells in the presence or absence of antigen. CD4 $\mathrm{T}$ cells were recovered at 16 - and 48 -h time points to examine activation by surface staining and cytokine production was detected in the supernatant. In some assays, DCs were also incubated with
$10 \mu \mathrm{g} \mathrm{ml}^{-1}$ neutralizing anti-TLR5 monoclonal antibody (Invivogen, San Diego, CA) for 60 min before addition of flagellin and SM1 T cells.

Flow cytometric analysis. DC subsets were characterized using antibodies specific for CD11c, CD11b, CD8 $\alpha, \mathrm{CD} 103, \mathrm{DEC} 205$ (CD205), and CD4 (eBiosciences, San Diego, CA). T-cell activation was examined using antibodies specific for CD4, CD90.1, CD69, CD25, CD44, and CD62L (eBiosciences). Cells were analyzed using a BD Fortessa and data analyzed using FlowJo software (Treestar, Ashland, OR).

Measurement of IL-2 cytokine production by enzyme-linked immunosorbent assay. IL-2 cytokine production in 16 or $48 \mathrm{~h}$ culture supernatants was measured using an mIL-2 BD Opt EIA detection kit (BD Biosciences, San Jose, CA) according to the manufacturer's protocol. Absorbance was measured at $450 \mathrm{~nm}$ using a micro plate reader (Spectra Max M2, Molecular Devices, Sunnyvale, CA).

RNA isolation, complementary DNA synthesis, and reverse transcriptase-PCR. DCs were isolated from spleen and MLNs as described above. Total RNA was extracted using RNA Easy Mini Kit (Qiagen, Germantown, MD) and 100 ng RNA was reverse transcribed with taqman (Applied Biosystems, Grand Island, NY) following the manufacturer's protocol. TLR5-specific primers were purchased from Invivogen and $5 \mu \mathrm{l}$ of complementary DNA was used for the amplification from each sample. PCR conditions were exactly as suggested by Invivogen and PCR products were separated on a $2 \%$ agarose gel. Glyceraldehyde-3-phosphate dehydrogenase amplification was used as an internal control in each case.

Isolation of dendritic cells from LP and Peyer's patch. The small intestine was removed from at least 5 to 6 mice and washed twice with phosphate-buffered saline to remove fecal contents before Peyer's patches were excised. DCs were isolated from Peyer's patches using CD11c microbeads, as described for other lymphoid tissues above. Mucus was removed from the remaining intestinal tissue and washed with Hank's balanced salt solution before incubating in $25 \mathrm{ml}$ Hank's balanced salt solution containing $2 \mathrm{~mm}$ EDTA and 5\% fetal bovine serum for $10 \mathrm{~min}$ at $37^{\circ} \mathrm{C}$. Supernatant was removed and intestinal tissue cut into small pieces before incubation in a shaking incubator (150 r.p.m.) at $37^{\circ} \mathrm{C}$ for $1 \mathrm{~h}$ in $50 \mathrm{ml}$ of RPMI- 1640 containing $5 \%$ fetal bovine serum, $1 \mathrm{mg} \mathrm{ml}^{-1}$ Collagenase (Life technologies, Invitrogen, Grand Island, NY), $1 \mathrm{mg} \mathrm{ml}^{-1}$ dispase (Life Technologies, Invitrogen), and $40 \mu \mathrm{g} \mathrm{ml}^{-1}$ DNaseI (Roche Diagnostics, Indianapolis, IN). Tissue was centrifuged and pellets were resuspended in EHAA (Eagle Hank's amino acids) media containing $10 \mathrm{~mm}$ EDTA for $5 \mathrm{~min}$ at $37^{\circ} \mathrm{C}$. Cells were then passed through a $40-\mu \mathrm{m}$ strainer and washed twice with MACS buffer before CD11c + DCs were enriched using $\mathrm{CD} 11 \mathrm{c}+$ microbeads.

Statistical analysis. Statistical analysis of data was accomplished using InStat (GraphPad Software, La Jolla, CA). Data were compared using unpaired $t$-test between groups and were considered significantly different with a $P$-value of $<0.05$. Two-way analysis of variance analysis was also used for statistical analysis where appropriate.

SUPPLEMENTARY MATERIAL is linked to the online version of the paper at http://www.nature.com/mi

\section{ACKNOWLEDGEMENTS}

We acknowledge helpful discussions with current and past members of the McSorley laboratory and the laboratory of $\operatorname{Dr} \mathrm{S}$. Way in completion of these experiments. This work was supported by grants from the National Institutes of Health Al073672 and Al055743.

\section{DISCLOSURE}

The authors declared no conflict of interest.

c) 2014 Society for Mucosal Immunology 


\section{REFERENCES}

1. Macnab, R.M. Genetics and biogenesis of bacterial flagella. Annu. Rev. Genet. 26, 131-158 (1992).

2. Hayashi, F. et al. The innate immune response to bacterial flagellin is mediated by Toll-like receptor 5. Nature 410, 1099-1103 (2001).

3. Gohda, J., Matsumura, T. \& Inoue, J. Cutting edge: TNFR-associated factor (TRAF) 6 is essential for MyD88-dependent pathway but not toll/IL-1 receptor domain-containing adaptor-inducing IFN-beta (TRIF)-dependent pathway in TLR signaling. J. Immunol. 173, 2913-2917 (2004).

4. Mizel, S.B. \& Bates, J.T. Flagellin as an adjuvant: cellular mechanisms and potential. J. Immunol. 185, 5677-5682 (2010).

5. Miao, E.A. et al. Cytoplasmic flagellin activates caspase-1 and secretion of interleukin 1 beta via Ipaf. Nat. Immunol. 7, 569-575 (2006).

6. Franchi, L. et al. Cytosolic flagellin requires Ipaf for activation of caspase-1 and interleukin 1 beta in salmonella-infected macrophages. Nat. Immunol. 7, 576-582 (2006).

7. Lightfield, K.L. et al. Critical function for Naip5 in inflammasome activation by a conserved carboxy-terminal domain of flagellin. Nat. Immunol. 9, 1171-1178 (2008)

8. Zhao, Y. et al. The NLRC4 inflammasome receptors for bacterial flagellin and type III secretion apparatus. Nature 477, 596-600 (2011).

9. McSorley, S.J., Ehst, B.D., Yu, Y. \& Gewirtz, A.T. Bacterial flagellin is an effective adjuvant for CD4 T cells in vivo. J. Immunol. 169, 3914 (2002).

10. Turley, C.B. et al. Safety and immunogenicity of a recombinant M2eflagellin influenza vaccine (STF2.4XM2e) in healthy adults. Vaccine 29, 5145-5152 (2011).

11. Taylor, D.N. et al. Induction of a potent immune response in the elderly using the TLR-5 agonist, flagellin, with a recombinant hemagglutinin influenzaflagellin fusion vaccine (VAX125, STF2.HA1 SI). Vaccine 29, 4897-4902 (2011).

12. Salazar-Gonzalez, R.M. \& McSorley, S.J. Salmonella flagellin, a microbial target of the innate and adaptive immune system. Immunol. Lett. 101, 117-122 (2005).

13. Cookson, B.T. \& Bevan, M.J. Identification of a natural T cell epitope presented by Salmonella-infected macrophages and recognised by Tcells from orally immunised mice. J. Immunol. 158, 4310-4319 (1997).

14. McSorley, S.J., Cookson, B.T. \& Jenkins, M.K. Characterization of CD4 + $\mathrm{T}$ cell responses during natural infection with Salmonella typhimurium. J. Immunol. 164, 986-993 (2000)

15. Bergman, M.A., Cummings, L.A., Alaniz, R.C., Mayeda, L., Fellnerova, I. \& Cookson, B.T. CD4 + -T-cell responses generated during murine Salmonella enterica serovar Typhimurium infection are directed towards multiple epitopes within the natural antigen FliC. Infect. Immun. 73, 7226-7235 (2005).

16. Cookson, B.T., Cummings, L.A. \& Rassoulian Barrett, S.L. Bacterial antigens elicit $\mathrm{T}$ cell responses via adaptive and transitional immune recognition. Curr. Opin. Microbiol. 4, 267-273 (2001).

17. Lodes, M.J. et al. Bacterial flagellin is a dominant antigen in Crohn disease. J. Clin. Invest. 113, 1296-1306 (2004).

18. Targan, S.R. et al. Antibodies to CBir1 flagellin define a unique response that is associated independently with complicated Crohn's disease. Gastroenterology 128, 2020-2028 (2005).

19. Dubinsky, M.C. et al. Serum immune responses predict rapid disease progression among children with Crohn's disease: immune responses predict disease progression. Am. J. Gastroenterol. 101, 360-367 (2006).

20. Jenkins, M.K. et al. In vivo activation of antigen-specific CD4 T cells. Annu. Rev. Immunol. 19, 23-45 (2001).

21. Means, T.K., Hayashi, F., Smith, K.D., Aderem, A. \& Luster, A.D. The Tolllike receptor 5 stimulus bacterial flagellin induces maturation and chemokine production in human dendritic cells. J. Immunol. 170, 5165-5175 (2003).

22. Salazar-Gonzalez, R.M. et al. Salmonella flagellin induces bystander activation of splenic dendritic cells and hinders bacterial replication in vivo. J. Immunol. 179, 6169-6175 (2007).

23. Uematsu, S. \& Akira, S. Immune responses of TLR5 $(+)$ lamina propria dendritic cells in enterobacterial infection. J. Gastroenterol. 44, 803-811 (2009).
24. Uematsu, S. et al. Detection of pathogenic intestinal bacteria by Toll-like receptor 5 on intestinal CD11c + lamina propria cells. Nat. Immunol. 7, 868-874 (2006).

25. Bates, J.T., Uematsu, S., Akira, S. \& Mizel, S.B. Direct stimulation of trr5 $+/+$ CD11c + cells is necessary for the adjuvant activity of flagellin. J. Immunol. 182, 7539-7547 (2009).

26. McSorley, S.J., Asch, S., Costalonga, M., Rieinhardt, R.L. \& Jenkins, M.K. Tracking Salmonella-specific CD4 T cells in vivo reveals a local mucosal response to a disseminated infection. Immunity 16, 365-377 (2002).

27. Srinivasan, A., Foley, J., Ravindran, R. \& McSorley, S.J. Low-dose Salmonella infection evades activation of flagellin-specific CD4 T cells. J. Immunol. 173, 4091-4099 (2004).

28. Letran, S.E., Lee, S.J., Atif, S.M., Uematsu, S., Akira, S. \& McSorley, S.J. TLR5 functions as an endocytic receptor to enhance flagellin-specific adaptive immunity. Eur. J. Immunol. 41, 29-38 (2011).

29. Letran, S.E. et al. TLR5-deficient mice lack basal inflammatory and metabolic defects but exhibit impaired CD4 T cell responses to a flagellated pathogen. J. Immunol. 186, 5406-5412 (2011).

30. Miao, E.A., Andersen-Nissen, E., Warren, S.E. \& Aderem, A. TLR5 and Ipaf: dual sensors of bacterial flagellin in the innate immune system. Semin. Immunopathol. 29, 275-288 (2007).

31. Vijay-Kumar, M., Carvalho, F.A., Aitken, J.D., Fifadara, N.H. \& Gewirtz, A.T. TLR5 or NLRC4 is necessary and sufficient for promotion of humoral immunity by flagellin. Eur. J. Immunol. 40, 3528-3534 (2010).

32. Steinman, R.M. The dendritic cell system and its role in immunogenicity. Annu. Rev. Immunol. 9, 271-296 (1991).

33. Shortman, K. \& Liu, Y.J. Mouse and human dendritic cell subtypes. Nat. Rev. Immunol. 2, 151-161 (2002).

34. Cong, $\mathrm{Y}$. et al. CD4 $+\mathrm{T}$ cells reactive to enteric bacterial antigens in spontaneously colitic $\mathrm{C} 3 \mathrm{H} / \mathrm{HeJBir}$ mice: increased $\mathrm{T}$ helper cell type 1 response and ability to transfer disease. J. Exp. Med. 187, 855-864 (1998).

35. Lee, S.J. et al. Temporal expression of bacterial proteins instructs host CD4 T cell expansion and th17 development. PLoS Path. 8, e1002499 (2012).

36. Ada, G.L., Nossal, G.J., Pye, J. \& Abbot, A. Behaviour of active bacterial antigens during the induction of the immune response. I. Properties of flagellar antigens from Salmonella. Nature 199, 1257-1259 (1963).

37. Nossal, G.J., Ada, G.L. \& Austin, C.M. Behaviour of active bacterial antigens during the induction of the immune response. li. Cellular distribution of flagellar antigens labelled with iodine-131. Nature 199, 1259-1262 (1963).

38. Parish, C.R. Immune response to chemically modified flagellin. I. Induction of antibody tolerance to flagellin by acetoacetylated derivatives of the protein. J. Exp. Med. 134, 1-20 (1971).

39. Parish, C.R. Immune response to chemically modified flagellin. II. Evidence for a fundamental relationship between humoral and cell-mediated immunity. J. Exp. Med. 134, 21-47 (1971).

40. Iwasaki, A. \& Medzhitov, R. Toll-like receptor control of the adaptive immune responses. Nat. Immunol. 5, 987-995 (2004).

41. Kinnebrew, M.A. et al. Interleukin 23 production by intestinal CD103(+) $\mathrm{CD} 11 \mathrm{~b}(+)$ dendritic cells in response to bacterial flagellin enhances mucosal innate immune defense. Immunity 36, 276-287 (2012).

42. Yarovinsky, F., Kanzler, H., Hieny, S., Coffman, R.L. \& Sher, A. Toll-like receptor recognition regulates immunodominance in an antimicrobial CD4 + T cell response. Immunity 25, 655-664 (2006).

43. Mariathasan, S. et al. Differential activation of the inflammasome by caspase-1 adaptors ASC and Ipaf. Nature 430, 213-218 (2004).

44. Adachi, O. et al. Targeted disruption of the MyD88 gene results in loss of IL-1- and IL-18-mediated function. Immunity 9, 143-150 (1998).

45. Barnden, M.J., Allison, J., Heath, W.R. \& Carbone, F.R. Defective TCR expression in transgenic mice constructed using CDNA-based alpha- and beta-chain genes under the control of heterologous regulatory elements. Immunol. Cell. Biol. 76, 34-40 (1998).

46. Parish, C.R. Fluorescent dyes for lymphocyte migration and proliferation studies. Immunol. Cell. Biol. 77, 499-508 (1999). 